\title{
Downlink Resource Allocation for Autonomous Infrastructure-based Multihop Cellular Networks
}

\author{
Mahdi Shabany ${ }^{1}$ and Elvino S. Sousa ${ }^{2}$ \\ ${ }^{1}$ Department of Electrical Engineering, Sharif University of Technology, P.O. Box 11365-8639, Tehran, Iran \\ ${ }^{2}$ Edward S. Rogers Sr. Department of Electrical and Computer Engineering, University of Toronto, Toronto, ON, Canada M5S $3 G 4$
}

Correspondence should be addressed to Mahdi Shabany, mahdi@eecg.toronto.edu

Received 18 July 2008; Revised 7 December 2008; Accepted 15 February 2009

Recommended by Joerg Kliewer

Considering a multihop cellular system with one relay per sector, an effective modeling for the joint base-station/relay assignment, rate allocation, and routing scheme is proposed and formulated under a single problem for the downlink. This problem is then formulated as a multidimensional multichoice knapsack problem (MMKP) to maximize the total achieved throughput in the network. The well-known MMKP algorithm based on Lagrange multipliers is modified, which results in a near-optimal solution with a linear complexity. The notion of the infeasibility factor is also introduced to adjust the transmit power of base stations and relays adaptively. To reduce the complexity, and in order to analyze the underlying key factors in the system, the framework is restricted to a two-base-station two-relay system. In fact, the output of the proposed algorithm is the joint optimization of the routing path, and base-station selection to achieve the maximum total throughput in the system, which in conjunction with the proposed adaptive scheme leads to the implementation of the cell breathing via allocating the proper transmit power to the basestations and relays.

Copyright ( 2009 M. Shabany and E. S. Sousa. This is an open access article distributed under the Creative Commons Attribution License, which permits unrestricted use, distribution, and reproduction in any medium, provided the original work is properly cited.

\section{Introduction}

Future wireless systems will require the capability to support very large throughput in selected areas, according to the location dependent and dynamic user demand, rather than the capability to support uniform traffic and coverage throughout a large service area. Ultimately given the constraint on the available bandwidth, very large system capacities can only be obtained by going to smaller cells by devising techniques for the reduction of the intercell interference. One effective approach to do so is to utilize multihop cellular network architectures [1-7].

In multihop networks, transmissions from a base station (BS) to a terminal occur over a number of hops, where devices other than base stations/access points or enduser terminals act as repeaters. The repeaters can be user terminals with a special functionality for relaying messages in addition to acting as end-user equipment [8-10] (called Type I multihop in this paper), or they can be devices with functionality restricted to relaying messages not including the users (Type II multihop). In Type II multihop, repeaters can be designed with a simplified functionality, so that their cost can be significantly less than that of a regular BS or access point. With this approach and with the capability of being self-configuring, these relays can be deployed in an autonomous manner, so that the network infrastructure grows organically according to the local need for the capacity increase [11]. Therefore, in this paper, we focus on Type II multihop cellular networks.

When it comes to the utilized spectrum, multihop cellular networks fall into two main categories: (i) the repeaters can be designed to receive and transmit on the regular frequency division duplex (FDD) bands of a cellular system with the possible use of a time slot scheduling structure such that where repeaters receive in even numbered slots and transmit in odd numbered slots or (ii) an additional frequency band can be allocated for the use of transmissions from BSs to the repeaters and also for repeaterto-repeater transmissions. We refer to transmissions directed to repeaters, whether from the BS or from another repeater, 
as the forwarding traffic. We also refer to the transmission on the regular FDD bands as in-band forwarding, and the transmission on an additional unlicensed band as out-ofband forwarding. In-band forwarding is normally used in Type I multihop networks [12], whereas in Type II multihop networks, out-of-band forwarding is preferred. This is due to the fact that with in-band forwarding, the multihop structure results in a traffic bottleneck at the BS and at repeaters that are in the first hop. With out-of-band forwarding, which is the focus of this paper, the added spectrum alleviates this bottleneck problem and results in a much larger capacity per BS. The out-of-band approach also has the attractive feature that all terminals can be legacy terminals. With the proper design of the repeaters, the legacy terminals can easily operate in the multihop cellular network using the same protocols used in the single-hop cellular networks. In other words, the multihop architecture becomes transparent to the end-user terminals. In this case, the approach of adding repeaters in an autonomous manner and in a multihop structure becomes a scalable capacity enhancing technique for a regular cellular system.

We refer to the traffic over hops where the destination is an end-user terminal as access traffic as opposed to the forwarding traffic referred to above that involves a repeater as the receiver. Thus, the forwarding traffic utilizes the outof-band spectrum, whereas access traffic utilizes the in-band spectrum. The out-of-band approach essentially separates the forwarding and access traffic. In so doing, from the access traffic standpoint, the repeaters behave as if they are BSs. In the forward link of wireless systems, a general resource allocation scheme optimized both in time and frequency such as the one in next generation network (NGN) can be considered. Here, without loss of generality, we consider only a time domain scheduling (TDS) scheme, where over short time slots, all the transmitted bits are directed to a given terminal.

In this paper, we propose an architecture for a network utilizing the out-of-band transmission for the forwarding traffic along with TDS for the access traffic for the joint optimization of the degree of the multihop, routing path, and BS selection to achieve the maximum total throughput in the system. The architecture under consideration is restricted to a two-BS two-relay system in two adjacent sectors facing each other. Although this model seems limited in scope, studying the resource allocation in this context allows us to derive a significant insight into the network performance behavior with respect to some key parameters of interest. Moreover, this model can be extended to either the case where the adjacent sectors within a cell are involved in the resource allocation or the intrasector cooperation where all BSs and relays within a two-tier hexagonal cell configuration are considered together.

Multihop cellular systems have been proposed in [1$3,8,13$ ]. These systems have been shown to both improve the throughput and reduce the required total transmission power. However, there have been only few results clarifying how the self-configuring feature can improve the system capacity. Most current routing algorithms for multihop cellular systems $[8,9,14]$ use path loss between terminals

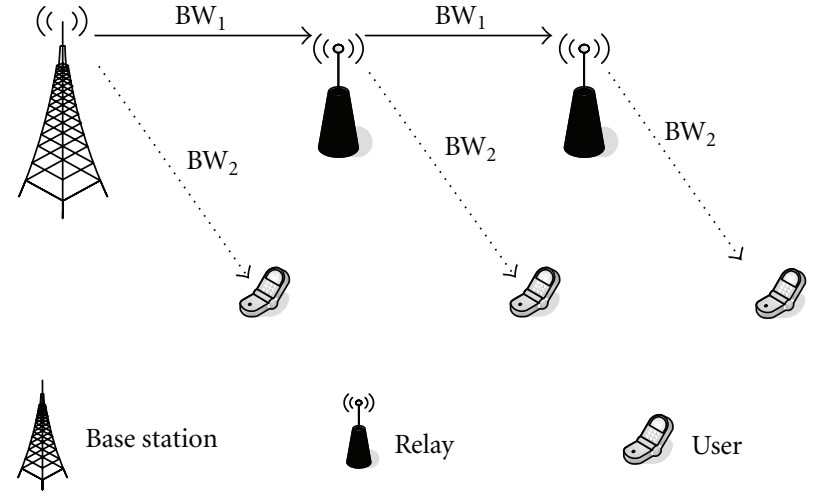

Figure 1: The proposed system model for the scalable selfconfiguring networks.

and transmitters as a metric to determine the routing. Although these approaches are easy to implement, they are not optimal and cannot manage the congested areas or cells. The proposed routing algorithm in [15] is based on the receiver interference, but the underlying proposed scheme does not optimize the total achieved network throughput. A centralized eigenvalue-based routing algorithm for multihop cellular networks is presented in [13]. Regardless of the substantial disparities in the system topology, the objective is to balance between signal to interference plus noise ratio (SINR) and the total network power consumption. Although [3] addressed the capacity of the multihop relaying with nonuniform traffic, the topology and the routing issues are not addressed in [3]. In [16], a multiple-layer access network that uses the hierarchical routing is proposed, which leads to a low-power architecture for the uplink transmission.

\section{System Model}

We consider a two-tier hexagonal cell configuration. Each cell has a specific geographical coverage area, which is divided to six sectors. The multihop system under consideration is realized by the insertion of one relay in each sector of a BS. Our proposed scalable network architecture is shown in Figure 1. There are three main elements in the network meaning: BSs, relays, and the users. Thus, there are three possible links between the BS and relays and terminals. Since the insertion of the relays should be transparent to users, the transmission from the relays and BSs to the users (access traffic) needs to be on the same frequency band, denoted by $f_{2}$ in Figure 1. In fact, the insertion of relays does not make any change to the network from the users' point of view. However, as justified in the introduction, the transmission from the BSs to the relays or between two relays (forwarding traffic) is of a different frequency band $\left(\mathrm{BW}_{1}\right)$ from the one used by the users in the cellular network $\left(\mathrm{BW}_{2}\right)$. This might be from an unlicensed frequency band, which makes them able to receive and transmit at the same time. This makes the architecture scalable as any other relay can be inserted in the system without any variation in the system topology. 


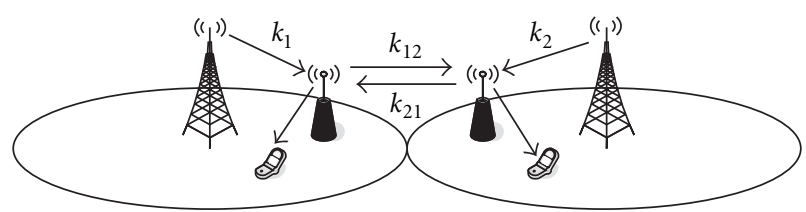

FIgURE 2: Two-BS two-relay system configuration.

In this paper, without loss of generality and for quantitative analysis of the system, we assume that each BS can coordinate only with its six neighboring BSs, and the maximum allowable number of hops is two. The system is shown in Figure 2, which shows two BS sectors facing each other. Therefore, in a two-BS two-relay system, there are four transmitters $i=1,2,3,4$, where $i=1(2)$ corresponds to the BS $1(2)$, and $i=3(4)$ denotes the relay $1(2)$, respectively. The fast power control, compensating the fast fading, is assumed to be done by its corresponding mechanism, and the open-loop power control, compensating the slow fading or shadowing, is performed every $T_{w}$ seconds. Note that different maximum power constraints for BSs and relays are considered. We assume that each user $j$ has a required average data rate $\left(\bar{R}_{j}\right)$. Our proposed scheme for the routing and resource allocation is performed every $T_{w}$ seconds, which is the length of a frame in time. The typical value of $T_{w}$ is 10 milliseconds in the universal mobile telecommunications system (UMTS) [17]. The proposed scheme in this paper can be thought of as a scheduler, determining the set of users that should be served within each time slot. Considering the TDS scheme, each user in the set is served within a fraction of the whole period by the maximum BS transmit power.

\section{Problem Definition}

Our main objective in this paper is to maximize the total network throughput in a multihop cellular system, while considering the power constraints of BSs and relays. In fact, to maximize the total network throughput, the optimum set of users and their corresponding routes to connect to the system should be determined. We assume that users do not have any limitation on their maximum received data rate, and the buffer size of relays is large enough. Based on the system in Figure 2, only two BSs, corresponding to two neighboring sectors, are involved in the resource allocation independent of the others. We assume that each relay covers $p(0 \leq p \leq 100)$ percentage of its corresponding sector area. The resource allocation problem is solved for a snapshot of the network whose length is $T_{w}$ seconds, so all the parameters defined hereafter belong to a snapshot of the system. Let us assume that there are $N$ users in the system and let $\mathbf{R}_{T}$ denote the system total achieved rate vector with a $1 \times(N+4)$ matrix as $\mathbf{R}_{T}=\left[r_{1}, r_{2}, r_{3}, r_{4}, r_{5}, \ldots, r_{N+4}\right]$, where $r_{1}, r_{2}, r_{3}$, $r_{4}$ are the allocated rates of the first BS, second BS, first relay, and second relay, respectively. The rest of the elements, $r_{5}, \ldots, r_{N+4}$, represent the rates of the users. The elements of $\mathbf{R}_{T}$ are positive if they represent the received rate and are negative if they show the transmitted rate. Due to the system's assumptions, $r_{i}>0$ for $i=5, \ldots, N+4$, as users cannot forward the access traffic to the others (Type II multihop).

Definition 1. $\mathbf{V}_{i, j}$ is a base vector whose $i$ th element is -1 and its $j$ th element is +1 and all the other elements are zero. (e.g., $\mathbf{V}_{1,3}=[-1,0,1,0, \ldots, 0]$ means that BS 1 transmits and relay 1 receives).

Definition 2. Vector $\mathbf{R}_{T}$ defined above is a system rate vector if and only if it can be written as a linear combination of $\mathbf{V}_{1, j} j \in\{3,5, \ldots, N+4\}, \mathbf{V}_{2, j} j \in\{4, \ldots, N+4\}, \mathbf{V}_{3, j} j \in$ $\{4, \ldots, N+4\}$, or $\mathbf{V}_{4, j} j \in\{3,5, \ldots, N+4\}$ with positive coefficients.

In fact, $\mathbf{R}_{T}$ simply represents the case, where every transmitter sends to only one receiver at each time slot (TDS). Here, the BSs are the transmitters, relays can be either transmitters or receivers, and the mobiles are always the receivers. Let $\mathbf{R}$ denote the optimal rate allocation vector at a specific time slot for a set of two neighboring sectors. Thus, given the time slot length, $T_{w}$, the optimal data allocation vector would be $\mathbf{R} T_{w}$. Since $\mathbf{R}$ is a system's total achieved rate vector, based on the definition of $\mathbf{R}_{T}$, it can be written as $\mathbf{R}=\left[-R_{T 1},-R_{T 2}, 0,0, R_{1}, R_{2}, \ldots, R_{N}\right]$, where $R_{T 1}$, and $R_{T 2}$ represent the total allocated data rate by BS 1 and 2, respectively. Moreover, $R_{1}, \ldots, R_{N}$ denote the total received data rate of $N$ users. This means that the total amount of transmitted and received data by relays are the same. Therefore, no data is buffered in relays at the end of each time slot (two zeros in the matrix). Note that no data buffering means that no data is buffered once the current time slot is over (i.e., at the end of each $T_{w}$ seconds, the relays are empty). However, data can be buffered in a relay during this period and be sent at the proper time before the time expires. Using the definition of the system's rate vector $(\mathbf{R})$ and the fact that the transmission at this rate happens during $T_{w}$ seconds, the total system's transmitted data vector can be written as follows:

$$
\begin{aligned}
\mathbf{R} T_{w}=\sum_{j=5}^{N+4}\{ & \tau_{1 j} R_{1 j} \mathbf{V}_{1, j}+\tau_{2 j} R_{2 j} \mathbf{V}_{2, j} \\
& +\tau_{3 j} R_{3 j}\left(\mathbf{V}_{1,3}+\mathbf{V}_{3, j}\right) \\
& +\tau_{4 j} R_{4 j}\left(\mathbf{V}_{2,4}+\mathbf{V}_{4, j}\right) \\
& +\tau_{5 j} R_{3 j}\left(\mathbf{V}_{2,4}+\mathbf{V}_{4,3}+\mathbf{V}_{3, j}\right) \\
& \left.+\tau_{6 j} R_{4 j}\left(\mathbf{V}_{1,3}+\mathbf{V}_{3,4}+\mathbf{V}_{4, j}\right)\right\}
\end{aligned}
$$

where $R_{i j}$ is the maximum data rate that transmitter $i$ (a BS or relay) can transmit to the receiver $j$ (users) while using its total allowable power, determined based on the hardware limitations. Moreover, $\tau_{i j},\left(\tau_{i j} \geq 0, \forall i, j\right)$ is the required time that transmitter $i$ has to consume to be able to support the average required data rate to user $j$ while transmitting by the maximum rate $R_{i j}$. Since each user $j$ is assumed to have a required average rate $\bar{R}_{j}$, its serving time, $\tau_{i j}$, should be large enough to be able to support this amount of data. Finally, $\tau_{5 j}$ and $\tau_{6 j}$ represent the amount of time during which relays 1 and 2 transmit the packets that are to be transmitted by two 
hops, respectively (Figure 3). The first (second) term in the above summation is corresponding to the case that user $j$ is served by BS 1(2), while the third (fourth) term corresponds to the case where user $j$ is served by BS 1(2) via relay 1(2). The last two terms are the cases where user $j$ is served by BS 1 (2) via two hops, respectively.

Because there is no diversity in the system, for each user, only one of the above terms is nonzero meaning that a user is served by a specific route at each time slot. This route determines the set of transmitters that are involved in forwarding the packets of that user and is fixed during each $T_{w}$ seconds. Therefore, the nonzero term determines both the transmitter to which the user is connected to and the routing path through which the packets are being forwarded. For instance, $\tau_{1 j} \neq 0$ means that user $j$ is served by BS 1 directly; $\tau_{3 j} \neq 0$ means that user $j$ gets its packets from BS 1 after being forwarded to relay 1 ; finally, $\tau_{6 j} \neq 0$ means that packets of user $j$ are forwarded from BS 1 to relay 1 then to relay 2 and eventually to user $j$, (see Figure 3 for all possible combinations).

The total power of BSs, $P_{\mathrm{BS}}$, is higher than that of relays, $P_{\mathrm{RLY}}$ (e.g., in our simulations, we used $P_{\mathrm{BS}}=L P_{\mathrm{RLY}}$, where $L=5$ ). This is because the relays are usually much smaller and less costly than BSs and can be deployed more easily at the required locations. Based on this fact and the location of BSs and relays (Figure 2), which is assumed to be symmetric, we can conclude that $R_{13} \cong R_{24}>R_{34}=R_{43}$. Our objective is to maximize the total downlink throughput in the set of two neighboring BSs, which leads to a suboptimal solution for the whole system. Therefore, using (1), the problem can be written as the following optimization problem:

$$
\max _{\tau_{i j}}\left\{\sum_{j=5}^{N+4}\left(\tau_{1 j} R_{1 j}+\tau_{2 j} R_{2 j}+\left(\tau_{3 j}+\tau_{5 j}\right) R_{3 j}+\left(\tau_{4 j}+\tau_{6 j}\right) R_{4 j}\right)\right\}
$$

$$
\begin{array}{ll}
\text { s.t. } & \sum_{j=5}^{N+4}\left(\tau_{1 j}+\frac{\tau_{3 j} R_{3 j}+\tau_{6 j} R_{4 j}}{R_{13}}\right) \leq T_{w}, \\
& \sum_{j=5}^{N+4}\left(\tau_{2 j}+\frac{\tau_{4 j} R_{4 j}+\tau_{5 j} R_{3 j}}{R_{24}}\right) \leq T_{w}, \\
& \sum_{j=5}^{N+4}\left(\tau_{3 j}+\tau_{5 j}+\frac{\tau_{6 j} R_{4 j}}{R_{34}}\right) \leq T_{w}, \\
& \sum_{j=5}^{N+4}\left(\tau_{4 j}+\tau_{6 j}+\frac{\tau_{5 j} R_{3 j}}{R_{34}}\right) \leq T_{w} .
\end{array}
$$

(Note that the above optimization problem can be generalized by considering a weighted summation. This would not change the problem formulation as the proposed suboptimal framework can also be extended in that case. In other words, the proposed scheme can be applied to any arbitrary weighted summation with different coefficients, appearing in the problem constraints in (3)-(6). Conceptually, the introduction of the weights can be used to achieve fairness, prioritize users, or optimize a more general cost function.
However, in this paper, the total achieved throughput is the objective cost function.)

The summation in (2) represents the total forwarded data to all the users during a time slot (e.g., $\sum_{j=5}^{N+4} \tau_{1 j} R_{1 j}$ denotes the total amount of data that is sent by BS 1). The constraints in (3)-(6) represent the time limitations during each time slot for BS 1 , BS 2 , relay 1 , and relay 2 , respectively. This implies that the total allocated time by a transmitter should be equal or less than the length of a time slot, $T_{w}$. For instance, the first term in (3) denotes the required time to support user $j$ when it is assigned directly to BS 1 . The second term is the amount of time that BS 1 should spend in order to support user $j$ via relay 1 , and finally, the last term corresponds to the amount of allocated time of BS 1 to support user $j$ via relay 1 and then relay 2 , respectively. We assume that relays can transmit and receive at the same time, and $\tau_{i j}$ is normalized by $T_{w}$ or simply $T_{w}=1$.

The optimization problem in (2) along with the constraints in (3)-(6) is NP-hard. Moreover, there is a dependency between the constraints in (3)-(6). Using an adaptive scheme proposed in Section 5, which converts the above four dependent constraints into six independent constraints, it is possible to map the above problem to a multidimensional multichoice knapsack problem (MMKP) (Section 4). Although MMKP is NP-hard, there are polynomial-time heuristic algorithms to solve it $[18,19]$. In our proposed adaptive scheme, each BS and relay reserves some portion of its total transmit power for forwarding the traffic of other relays or BSs. Referring to Figure 2, we assume that BS 1 reserves $k_{1} P_{\mathrm{BS}}$ for serving the packets that should be forwarded via relay 1 . Relay 1 also reserves $k_{12} P_{\mathrm{RLY}}$ for forwarding the packets that should be transmitted via relay 2 . These packets are transmitted by two hops. The same thing applies to relay 2 and BS 2 . We represent the reserved power of relay 2 and BS 2 by $k_{21} P_{\mathrm{RLY}}$ and $k_{2} P_{\mathrm{BS}}$, respectively. The values of $k_{1}, k_{2}, k_{12}, k_{21}$, all less than 1 , are adjusted every time slot based on the traffic profile of the BSs and relays so as to make the traffic load as much balanced as possible. In this paper, we refer to them simply as " $k$ parameters." In brief, the proposed adaptive scheme makes the constraints independent resulting in a balanced traffic load by adjusting the values of $k$ parameters. The details will be described in Section 5 .

\section{Mapping the Problem to MMKP}

Using the adaptive scheme, we show that the optimization problem in (2) can be mapped to an MMKP.

Proposition 1. Using the proposed adaptive scheme, the constraints in (3)-(6) can be written as

$$
\begin{aligned}
& \sum_{j=5}^{N+4} \tau_{1 j} \leq 1-k_{1}, \\
& \sum_{j=5}^{N+4} \tau_{2 j} \leq 1-k_{2},
\end{aligned}
$$




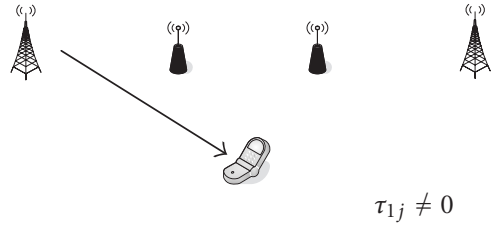

(a)

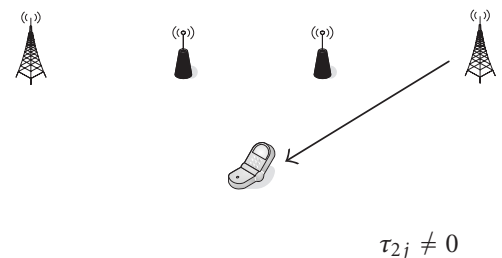

(b)

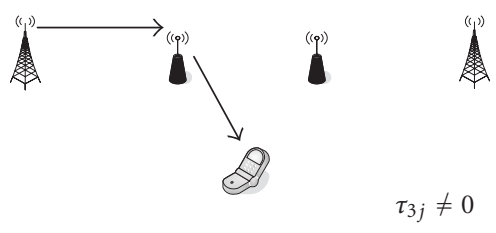

(c)

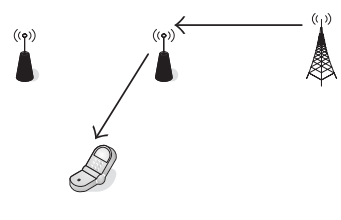

(d)

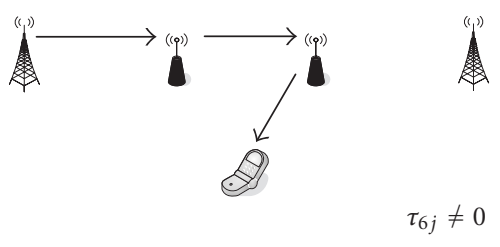

(e)

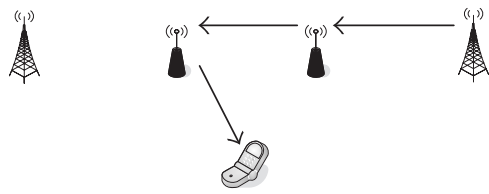

(f)

FIGURE 3: Possible routing paths for an arbitrary mobile within the network.

$$
\begin{gathered}
\sum_{j=5}^{N+4} \tau_{3 j} R_{3 j} \leq k_{1} R_{13}-k_{12} R_{34}, \\
\sum_{j=5}^{N+4} \tau_{4 j} R_{4 j} \leq k_{2} R_{24}-k_{21} R_{43}, \\
\sum_{j=5}^{N+4} \tau_{5 j} R_{3 j} \leq k_{21} R_{34}, \\
\sum_{j=5}^{N+4} \tau_{6 j} R_{4 j} \leq k_{12} R_{43} .
\end{gathered}
$$

Proof. See Appendix A.

Inequalities (7a)-(10b) are conceptually related to the different possible routing scenarios shown in Figures 3(a)3(f). Although these inequalities are time-based constraints, considering the TDS system, they also have power-based interpretations. In the time domain scheduling, during each time interval, the total power is allocated to a single user, while the rest of the users are inactive. The required time interval $\left(\tau_{1 j}, \tau_{2 j}, \ldots\right)$ is inversely proportional to the maximum deliverable data rate $\left(R_{i j}\right)$ in the TDS mode, and $R_{i j}$ is proportional to the total available transmit power. Therefore, more available power means smaller required time interval to serve a specific user. Thus, the available resource can be thought as either the time or power. For example, constraints in (7a) and (7b) are related to the maximum power limits of BSs 1 and 2, considering the reserved portion for the packet forwarding to relays 1 and 2 , respectively. The constraint in (8) takes into account the limitation on the transmitted power of the relay 1 , which is limited by the amount of power assigned to it by BS $1\left(k_{1} P_{\mathrm{BS}}\right)$ minus the portion of its power reserved for relay $2\left(k_{12} P_{\mathrm{RLY}}\right)$. Inequality (9) is the same constraint as (8) but for relay 2. Finally, constraints in (10a) and (10b) show the limitation on the amount of data that can be sent by each BS by forwarding via two relays due to the limited amount of allocated power for this purpose by relays $\left(k_{21} P_{\mathrm{RLY}}\right.$ and $\left.k_{12} P_{\mathrm{RLY}}\right)$. The values of $k_{1}, k_{2}, k_{12}, k_{21}$ should be adjusted based on the traffic of BSs and relays. Note that the $k$ parameters should be chosen such that $k_{1} R_{13}-k_{12} R_{34} \geq 0$ and $k_{2} R_{24}-k_{21} R_{43} \geq 0$. These two conditions correspond to the right hand-side of inequalities in (8) and (9) meaning that relays cannot forward more than the amount that is allocated by BSs. The constraints in (7a)(10b) can be represented in a general form of $\sum_{j=5}^{N+4} a_{i j} x_{j} \leq C_{i}$ for all $i \in\{1, \ldots, 6\}$, where $x_{j}$ is 1 when user $j$ is assigned to the network and 0 otherwise,

$$
a_{i j}= \begin{cases}\tau_{i j} & \text { if } i=1,2, \\ \tau_{i j} R_{i j} & \text { if } i=3,4, \\ \tau_{5 j} R_{3 j} & \text { if } i=5, \\ \tau_{6 j} R_{4 j} & \text { if } i=6,\end{cases}
$$

$C_{i}=\left(1-k_{1}\right),\left(1-k_{2}\right),\left(k_{1} R_{13}-k_{12} R_{34}\right),\left(k_{2} R_{24}-\right.$ $\left.k_{21} R_{43}\right),\left(k_{21} R_{34}\right)$, and $\left(k_{12} R_{43}\right)$ for $i=1, \ldots, 6$, respectively. Assignment of a user to the network implies that the user is being served during the current time slot.

Using Proposition 1, the optimization problem in (2) along with its constraints (7a)-(10b) can be mapped to an MMKP, an extended version of Knapsack problem (KP). In MMKP, there is an $M$-dimensional knapsack with $M$ total allowable volumes of $W_{1}, W_{2}, \ldots, W_{M}$. Furthermore, there are $N$ groups of items. Group $j$ has $n_{j}$ items. Each item has a value and an $M$-dimensional volume corresponding to knapsack's $M$ dimensions. The objective of the MMKP is to pick up exactly one item from each group to maximize the total value of the selected items, which subject to the volume constraints of knapsack's dimensions.

The mapping of (2) to an MMKP is as follows. We consider $M$ knapsacks (here $M=6$ ) presented by (7a)(10b) plus an auxiliary knapsack as "one knapsack" with $M+1$ dimensions, where the total allowable volume of dimension $i$ is $C_{i}$. Moreover, $C_{M+1}$ corresponding to the resource constraint of auxiliary knapsack is set to zero. Each 
user is considered as a group, which has $n_{j}$ (here $M+1$ ) items. All items of user $j$ except the $(M+1)$ th item have an equal value, which is the average required rate of that user. The value of the last item is always zero. The $k$ th item of $j$ th user requires an $M$-dimensional volume, which is defined as $A_{i j k}=a_{i j}$ if $i=k \neq M+1$ and zero otherwise.

This ensures that item $k$ of any group, that corresponds to knapsack $k$, can only be assigned to knapsack $k$. Therefore, if item $\hat{k}$ of group $j$ is selected in the optimal solution, it means that user $j$ has been assigned to the knapsack $\hat{k}$, its corresponding achieved throughput is $\bar{R}_{j}$, and the amount of resource it requires from the knapsack $\hat{k}$ is $a_{\hat{k} j}$. We have to choose exactly one item from each group meaning that each user can be assigned to at most one knapsack. On the other hand, by the definition of MMKP, we have to choose exactly one item from each group. However, the selection of all users is not feasible at all the times. Therefore, if user $j$ does not exist in the optimal solution, it means that its last item whose corresponding value and volumes are zero has been selected. This indirectly implies that user $j$ has not been assigned to the network.

Based on the above discussion, we can rewrite the optimization problem using (2) and $A_{i j k}$ as

$$
\begin{gathered}
\max _{x_{k j}}\left(\sum_{j=1}^{N} \sum_{k=1}^{M+1} x_{k j} \bar{R}_{j}\right), \\
\text { s.t. } \sum_{j=1}^{N} \sum_{k=1}^{M+1} x_{k j} A_{i j k} \leq C_{i} \quad \forall i \in\{1, \ldots, M\}, \\
\sum_{k=1}^{M+1} x_{k j}=1 \quad \forall j \in\{1, \ldots, N\}, x_{k j} \in\{0,1\},
\end{gathered}
$$

where $x_{k j}$ is one when the item $k$ of user $j$ is selected.

Because of the NP-hardness of the MMKP, exhaustive search algorithms such as branch-and-bound [20] with the globally optimal solutions are too time-consuming and can only be applied to the very small problems. The computational complexity of these algorithms is $O\left(2^{M^{2} N}\right)$. However, several heuristic algorithms have been proposed such as those in $[18,19]$, which are polynomial-time suboptimal algorithms. In this paper, we use the modified version of the algorithm presented in [18], which is based on the Lagrange multipliers. Numerical results comparing the performance of the suboptimal methods, and the branchand-bound method (Section 6) justifies the use of this heuristic algorithm. Here, for brevity of the discussion, we briefly outline the theory of Lagrange multipliers and the algorithm used to solve the MMKP based on the current notations in this section.

Theorem 1. Let $\lambda_{1}, \lambda_{2}, \ldots, \lambda_{M}$ be $M$ nonnegative Lagrange multipliers, and let $x_{k j}^{*}$ be the solution of

$$
\max _{x_{k j}}\left\{\left(\sum_{j=1}^{N} \sum_{k=1}^{M} x_{k j} \bar{R}_{j}\right)-\sum_{i=1}^{M} \lambda_{i} \sum_{j=1}^{N} \sum_{k=1}^{M+1} x_{k j} A_{i j k}\right\} .
$$

Then, the binary variables $x_{k j}^{*}$ are also the solution to

$$
\begin{gathered}
\max _{x_{k j}}\left(\sum_{j=1}^{N} \sum_{k=1}^{M} x_{k j} \bar{R}_{j}\right), \quad x_{k j} \in\{0,1\}, \\
\sum_{j=1}^{N} \sum_{k=1}^{M+1} x_{k j} A_{i j k} \leq \sum_{j=1}^{N} \sum_{k=1}^{M+1} x_{k j}^{*} A_{i j k}, \quad \forall i \in\{1, \ldots, M\} .
\end{gathered}
$$

Proof. See [21].

According to this theorem, the solution to the unconstrained optimization problem (15) is also the solution to the constraint optimization problem (16), which is the MMKP in (13) with the constraint values $C_{i}$ replaced by $\sum_{j=1}^{N} \sum_{k=1}^{M+1} x_{k j}^{*} A_{i j k}$. Therefore, if the multipliers $\lambda_{i}$ are known, the optimization problem is easily solved. This is because that (15) can be written as

$$
\max _{x_{k j}}\left\{\sum_{j=1}^{N} \sum_{k=1}^{M}\left(\bar{R}_{j}-\sum_{i=1}^{M} \lambda_{i} A_{i j k}\right) x_{k j}\right\},
$$

which implies that the solutions are

$$
x_{k j}^{*}= \begin{cases}1 & \text { if } \bar{R}_{j}-\sum_{i=1}^{M} \lambda_{i} A_{i j k}>0, \\ 0, & \text { otherwise. }\end{cases}
$$

Therefore, the only step to do is to compute the Lagrange multipliers $\lambda_{i}$. It is worth noting that if these multipliers be computed such that the terms $C_{i}-\sum_{j=1}^{N} \sum_{k=1}^{M} x_{k j}^{*} A_{i j k}$ are nonnegative, the solution is feasible. The heuristic algorithm based on the Lagrange multipliers, which produces suboptimal values for $\lambda_{i}$ and $x_{k j}$ simultaneously is shown in Algorithm 1. The algorithm starts with the most valuable item of each user $j$ as the selected item $\left(\hat{K}_{j}\right)$, and the Lagrange multipliers initialized to zero such that the constraints in (14) and (19) are satisfied. In general, however, the volume constraints will now be violated. The initial choice of the selected items is revised to obey the volume constraints by repeatedly improving the most violated constraint, $\widehat{I}$, as shown in Algorithm 1.

Consider the users whose selected items correspond to the BS $\hat{I}$ (i.e., $\left\{j \mid \hat{K}_{j}=\hat{I}\right\}$ ). For each item $k$ of these users, the increase $\Delta_{k j}$ in multiplier $\lambda_{\hat{I}}$ that results from exchanging the selected item of group $j$ is computed. Eventually, the item $K^{*}$ of user $J^{*}$, causing the least increase of multiplier $\lambda_{\hat{I}}$, is chosen for exchange. This choice minimizes the widening of the gap between the optimal solution characterized by $C_{i}-$ $\sum_{j=1}^{N} \sum_{k=1}^{M} x_{k j}^{*} A_{i j k}$ and the solution returned by the MMKP algorithm. The process is repeated until for each user an item has been selected such that the volume constraints are satisfied. Since each user has always an item whose value and the $M$-dimension volume is zero, the solution is always feasible.

After completion of DROP phase, there may be some space left in the knapsack. This space may be utilized to improve the solution by replacing some selected items 


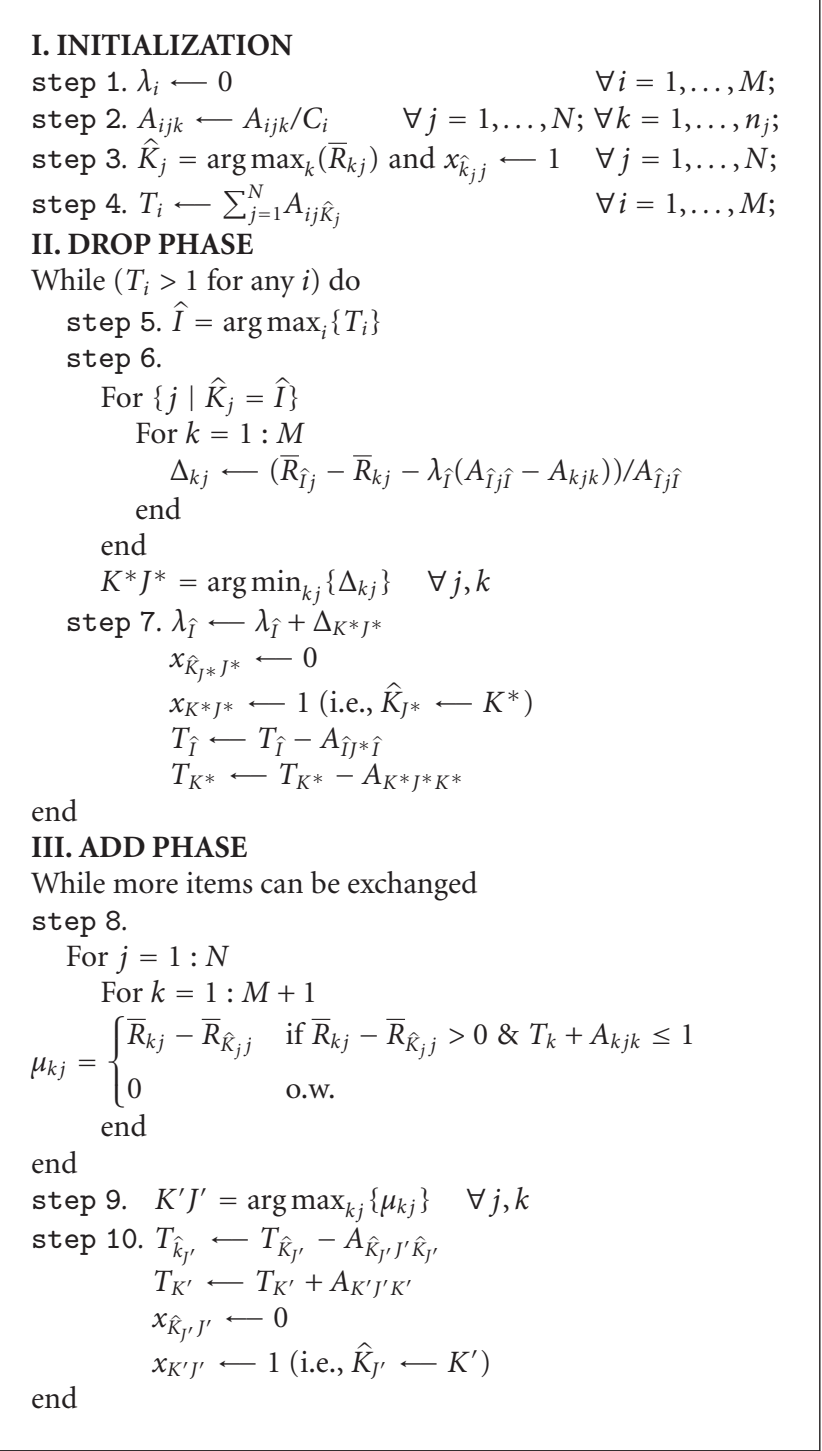

Algorithm 1: MMKP algorithm for resource allocation in multihop cellular.

with more valuable ones. Therefore, in $A D D$ phase of the algorithm, each item $k$ of every user $j$ is checked against the selected item of that user $\left(\widehat{K}_{j}\right)$. It is tested to see if item $k$ is more valuable than the selected item and if $k$ can replace the selected item without violating the volume constraints. Among all exchangeable items, the item $K^{\prime}$ of user $J^{\prime}$ causing the largest increase of the knapsack value is exchanged with the selected item of that user $\left(\hat{K}_{J^{\prime}}\right)$. This process is repeated until no more exchanges are possible. The resulting solution comprised of the selected items is feasible, and even optimal, if $\sum_{j=1}^{N}\left(\lambda_{i} C_{i}-\sum_{j=1}^{N} \sum_{k=1}^{M} x_{k j}^{*} A_{i j k}\right)=0$.

Note that due to the equality between the values of different items of each user $j, \bar{R}_{j}$, except its last item, we have to make a tiny modification to be able to apply the algorithm. One simple way would be adding a very small value but different to every item of a user. The last item should be kept zero. Let us denote the value of $k$ th item of $j$ th user by $\bar{R}_{k j}=\bar{R}_{j}+\varepsilon_{k j}$ for $k \in\{1, \ldots, M\}$, where $\varepsilon_{k j}$ is a very small value (e.g., $\varepsilon_{k j}=0.01$ in our simulations) but different for every item of a user. This modification is necessary when we are calculating the amount of increasing $\Delta_{k j}$ of the Lagrange multiplier corresponding to the most violated constraint, $\widehat{I}$, in Drop phase of the algorithm.

Theorem 2. The maximum difference between the total achieved throughput using the above suboptimal algorithm and the globally optimal solution is $\sum_{i=1}^{M} \lambda_{i}\left(C_{i}-\sum_{j=1}^{N} \sum_{k=1}^{M+1} x_{k j}^{*} A_{i j k}\right)$, where $x_{k j}^{*}$ is the output of the heuristic algorithm.

Proof. See Appendix B.

Based on Theorem 2, the solution is optimal if $\sum_{i=1}^{M} \lambda_{i}\left(C_{i}-\sum_{j=1}^{N} \sum_{k=1}^{M+1} x_{k j}^{*} A_{i j k}\right)=0$ (i.e., the case whereby error is zero). Numerical results in Section 6 show that most of the times of this gap is negligible. Therefore, the result is a good approximation of the globally optimal solution.

4.1. Computational Complexity. The following proposition indicates that the proposed algorithm has a polynomial-time computational complexity.

Proposition 2. The heuristic algorithm proposed in Algorithm 1 has a maximum computational complexity of $O\left(N^{2} M^{3}\right)$.

Proof. Step 1 has the complexity order of $O(M)$, and step 2 to step 4 have the complexity order of $O(N M)$. In the while loop, step 5 and step 7 have the complexity order of $O(M)$ and $O(1)$, respectively. In step 6 for each of $N$ users, there are at most $M$ nonselected routing paths, thus for each user, the maximum complexity order is $M$. Since there is one iteration for each potential path associated with each user, the total complexity order of step 6 is $O\left(N M^{2}\right)$. In every iteration of step 6 , one assigned path is removed from one user, thus, in the worst case, the while loop in the DROP phase is executed NM times. Therefore, the overall complexity order for the execution of the while loop of the DROP phase is $O\left(N^{2} M^{3}\right)$, where we also assume that $N \gg$ $M$. In the ADD phase, the complexity order of step 9 and step 10 is $O(N M)$ and $O(1)$, respectively. In step 8, for each of the $N$ users, at most $M$ nonselected paths at most are considered. Each computation has a complexity of $M$. There is one iteration for each knapsack, resulting in the complexity order of $O\left(N M^{2}\right)$ for step 8. Since for each user there are, at most, $M$ potential knapsacks, which could have higher rate than the assigned one, the outer while loop of the ADD phase algorithm is executed at most $N M$ times. This gives an overall complexity of $O\left(N^{2} M^{3}\right)$ for the ADD phase. Thus, the overall computational complexity is $O\left(N^{2} M^{3}\right)$.

\section{Adaptive Scheme to Adjust $k$ Parameters}

In this section, we propose a scheme for adjusting $k$ parameters to make the resource allocation scheme 
TABLE 1: Adjustment of the $k$ parameters.

\begin{tabular}{|c|c|c|c|c|c|}
\hline \multicolumn{2}{|c|}{ Two highest $Q$} & \multicolumn{4}{|c|}{$k$ values adjustments } \\
\hline$\overline{Q_{1}}$ & $\left(Q_{3}\right.$ or $\left.Q_{5}\right)$ & $k_{1} \downarrow$ & $k_{12} \downarrow$ & $k_{2} \uparrow$ & $k_{21} \uparrow$ \\
\hline$Q_{1}$ & $\left(Q_{4}\right.$ or $\left.Q_{6}\right)$ & $k_{1} \downarrow$ & $k_{12} \uparrow$ & $k_{2} \uparrow$ & $k_{21} \downarrow$ \\
\hline$Q_{1}$ & $Q_{2}$ & $k_{1} \downarrow$ & $k_{12} \uparrow$ & $k_{2} \downarrow$ & $k_{21} \downarrow$ \\
\hline$Q_{2}$ & $\left(Q_{3}\right.$ or $\left.Q_{5}\right)$ & $k_{1} \uparrow$ & $k_{12} \downarrow$ & $k_{2} \downarrow$ & $k_{21} \uparrow$ \\
\hline$Q_{2}$ & $\left(Q_{4}\right.$ or $\left.Q_{6}\right)$ & $k_{1} \uparrow$ & $k_{12} \uparrow$ & $k_{2} \downarrow$ & $k_{21} \downarrow$ \\
\hline$\left(Q_{4}\right.$ or $\left.Q_{6}\right)$ & $\left(Q_{3}\right.$ or $\left.Q_{5}\right)$ & $k_{1} \uparrow$ & $k_{12} \downarrow$ & $k_{2} \uparrow$ & $k_{21} \downarrow$ \\
\hline
\end{tabular}

self-adaptive to the system's traffic profile. The idea of adjusting the values of $k$ parameters comes from the fact that the maximum utilization of resources is obtained when there is cooperation between transmitters (BSs and relays). Let us assume that at time slot $n-1$, we have solved the problem (12) with constraints in (13)-(14). Thus, we know the best suboptimal assignments of users to the relays and BSs at this time slot. Based on the given assignments, we can define the infeasibility factors. The infeasibility factor of transmitter $i$ at time slot $n$ is defined as

$$
Q_{i}(n)=\sum_{j=5}^{N+4} \frac{a_{i j}(n) x_{j}(n)}{C_{i}}
$$

which represents the ratio of the amount of the allocated resource to the available resource of transmitter $i$ at time slot $n$. Remember that $i=1(2)$ corresponds to BS $1(2), i=3,5$ denotes the relay 1 , and $i=4,6$ corresponds to the relay 2 . Parameter $Q_{i}(n)$ shows the amount of the allocated resource of the knapsack $i$ at time slot $n$. Based on these infeasibility factors at each time slot, we can decide on the values of $k$ parameters in the next time slot. One can think of step-based variations in which, at each time slot, the $k$ parameters are increased or decreased by a specific predetermined value, which is the strategy used in our simulations. Table 1 shows all the different possible scenarios and their corresponding actions that need to be taken. The tables shows the two highest infeasibility factors as the base of the decision. For instance, if $Q_{1}$ and $Q_{3}$ are the two highest factors, the value of the $k_{1}$ should be decreased to release more resources to the BS1. At the same time $k_{12}$ should be reduced to give more resources to relay 1 . On the other side $k_{2}$ and $k_{21}$ may increase to take care of the possible overload of BS1 and relay1. The other scenarios can be explained in the same way.

\section{Simulation Results}

We consider a two-tier hexagonal cell configuration with a wrap-around technique. A universal mobile telecommunication system, with a fast power controller running at 1500 updates per second, is simulated. The total number of 19 cells with cell radius $1000 \mathrm{~m}$ and BTS transmit power of $10 \mathrm{~W}$ were considered. Moreover, the required $E / I$ for users is $-13 \mathrm{~dB}$, thermal noise density is $-174 \mathrm{dBm} / \mathrm{Hz}$, and propagation loss exponent is 4 . We focus on the central cell as the BS 1 and one of its neighbors as the BS 2 along with their

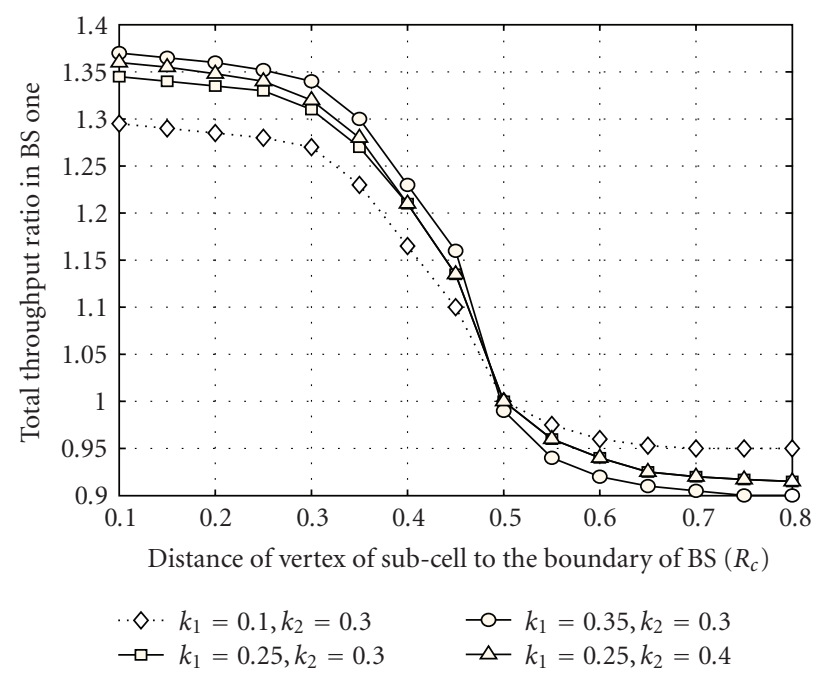

FIGURE 4: The ratio of the achieved throughput with relaying to the case without relaying in BS 1 versus the distance of the subcell to the BS for different values of $k_{1}$.

corresponding sectors. Hereafter, we call them couple BSs and couple sectors, respectively.

6.1. Throughput Versus Relay Location. First, we consider a two-BS two-relay set including the neighboring sectors of BS 1 and BS 2 with 70 users distributed nonuniformly through the BSs' coverage area as follows. Ten users were distributed randomly and uniformly throughout both cells (BS 1 and BS 2). Then, 60 users were distributed nonuniformly in BS 1 within a small hexagonal, called subcell, with radius $R_{c} / 10$, where $R_{c}$ is the radius of the cells. The maximum number of intermediate relays was set to 1 . The distance of subcell to $\mathrm{BS}$ is changed from $9 R_{c} / 10$ to $2 R_{c} / 10$. The ratio of the achieved throughput in cell 1 for the case with relays to the case without relays for different values of $k$ parameters is shown in Figure 4. In this case, we set $k_{12}=k_{21}=0$ (i.e., at most one relaying), $k_{2}=0.3$ and we changed the value of $k_{1}$. It is seen that when users are on the cell boundaries, our scheme outperforms greatly the case with no relays. However, by approaching the users toward the BS, our scheme's result approaches to the case where there is no relay, which is expected. The reason is that for users that are closer to the BS the chance of relaying is small. This concept is seen for the case where the distance between the center of the subcell and the BS 1 is less than $0.5 R_{c}$. Moreover, if the subcell approaches further to the BS, the result is degenerated and gets even worse than the case with no relays. The reason is that, in this case, the BS allocates a special portion of its power to the relay, which has a tiny role in supporting the users that are close to the BS. Therefore, the higher the value of $k_{1}$, the worse the results. This result indirectly implies the fact that the $k$ parameters $\left(k_{1}\right.$ here) should be adjusted properly based on the load of every transmitter and the traffic pattern of the users. Moreover, the ratio of the achieved throughput for different locations of the sub-cell for the case where both $k_{1}$ and $k_{2}$ change is also shown in Figure 4. It 
is seen that the increase in $k_{2}$ just affects those cases where the location of the subcell is close to the cell boundary. The reason is that by adjusting the $k_{1}^{\prime}$ more users are supported by BS 2 via relay 2 .

6.2. Intracell Relaying Versus Intercell Relaying. The ratio of the total achieved throughput in cell 1 for the case where there is at most one-relay forwarding option (intra-cell) to the case with two-relay forwarding (intercell) versus the location of the sub-cell for different values of $k_{21}$ is shown in Figure 5. As it is seen, the whole system performance is improved. The location of unity gain is also shifted closer to the BS. As $k_{21}$ increases, the result gets better. This is because some users are supported by BS 2 via relay 2 and then by relay 1 , respectively (i.e., two-relay forwarding). Moreover, as the subcell approaches further to the BS further this gain decreases.

6.3. Adaption to the User Topology and Number of Users. Figure 6 shows the throughput ratio of both cells, defined as the ratio of transmitted data over the required data versus the number of users in cell 2, which are distributed within a subcell under the relay 2 . It clearly shows that depends on the load of the BS 2, different values for $k_{21}$ needs to be used. For example, for 10 users, $k_{21}=0.2$ is the best choice, while for 14 users we have to switch to $k_{21}=0.4$. In this simulation, the number of users in BS 1 was set to 40 , which were distributed uniformly throughout the cell, $k_{1}=0.3, k_{12}=0$, and $k_{2}=0.3$.

Moreover, 60 users were distributed non-uniformly through the two-BS two-relay set such that half of the users were located too close to the relay 1 . The value of $k$ parameters was set initially to $k_{1}=0.15, k_{12}=0.5$, $k_{2}=0.3$, and $k_{21}=0.1$. The variations of these parameters were investigated in the four consecutive time slots. We assumed quantized variations where at each time slot, the $k$ parameters can increase or decrease at most 0.05 of a unit. The result is shown in Figure 7, where the horizontal axis shows the time, and the vertical axis shows the $k$ parameters. The values of the $k$ parameters were set improperly initially to be able to see the performance of our adaptive scheme in conjunction with the knapsack problem. As it was expected, the values of the $k$ parameters were adaptively adjusted such that the load becomes balanced between the transmitters. This means that the BS 1 allocated more power to the relay 1 . The values of $k_{1}$ and $k_{12}$ changed more compared to $k_{2}$ and $k_{21}$ as they are directly related to the relay 1 . Since the relay 1 is the most congested transmitter almost in all time slots, based on the adaptive scheme in Section 5, $k_{1}$ and $k_{12}$ should increase and decrease, respectively, in the consecutive time slots in order to provide more available resources to the relay 1 .

The ratio of the total achieved throughput with relays to that of without relays for the above four consecutive time slots was calculated. The ratio of the achieved throughput shows an increase from 1.04 at $T=0$ to $1.14,1.25,1.31$, and 1.34 at $T=1, T=2, T=3$, and $T=4$, respectively.

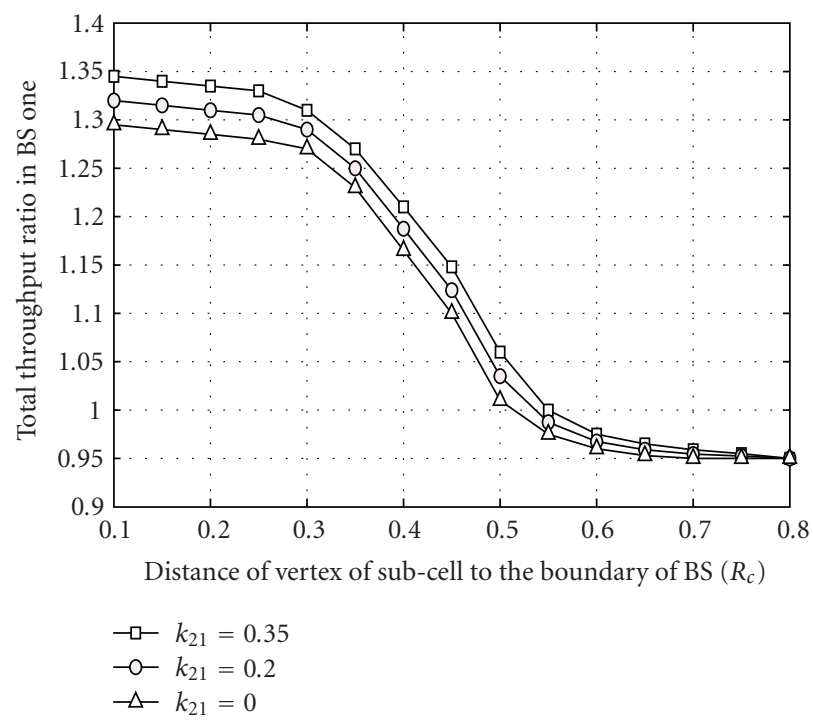

FIgURE 5: The ratio of the achieved throughput with relaying to the case without relaying in BS 1 for one-relaying and two-relaying cases versus the distance of the sub-cell to the BS for different values of $k_{21}$.

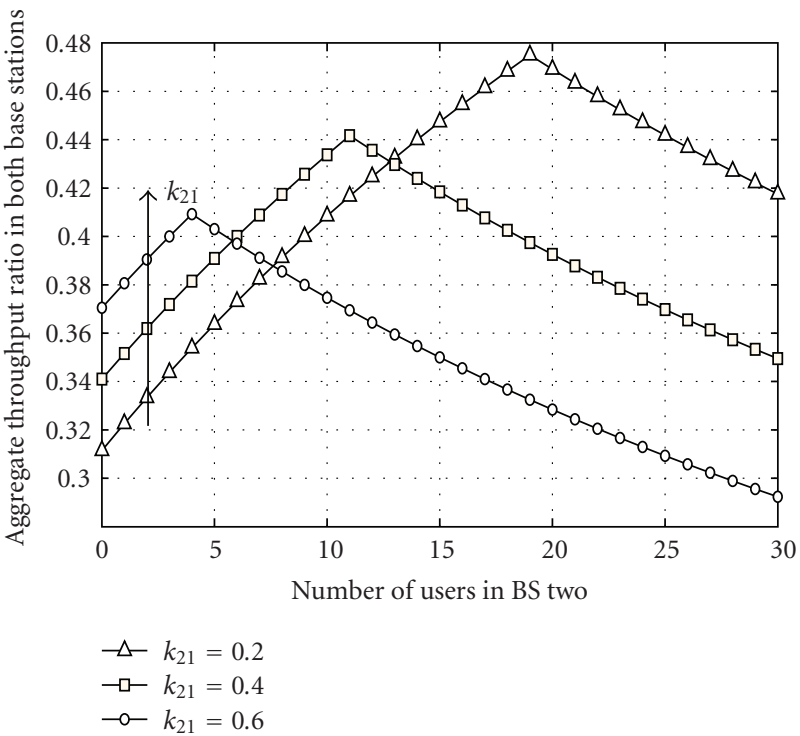

FIGURE 6: The aggregate throughput ratio in both base-stations versus the number of added users in BS 2 for different values of $k_{21}$.

This result clearly shows that the adjustment of $k$ parameters results in a better performance.

Figure 8 shows the variation of infeasibility factors due to the adjustments of $k$ parameters. The values of these factors are different at the first time slot because the values of $k$ parameters are not properly chosen. The difference in infeasibility factors is translated to difference in the load levels of knapsacks. By adjusting the $k$ parameters, however, as it is seen in the figure, the values of infeasibility factors approach to each other which mean that the load 

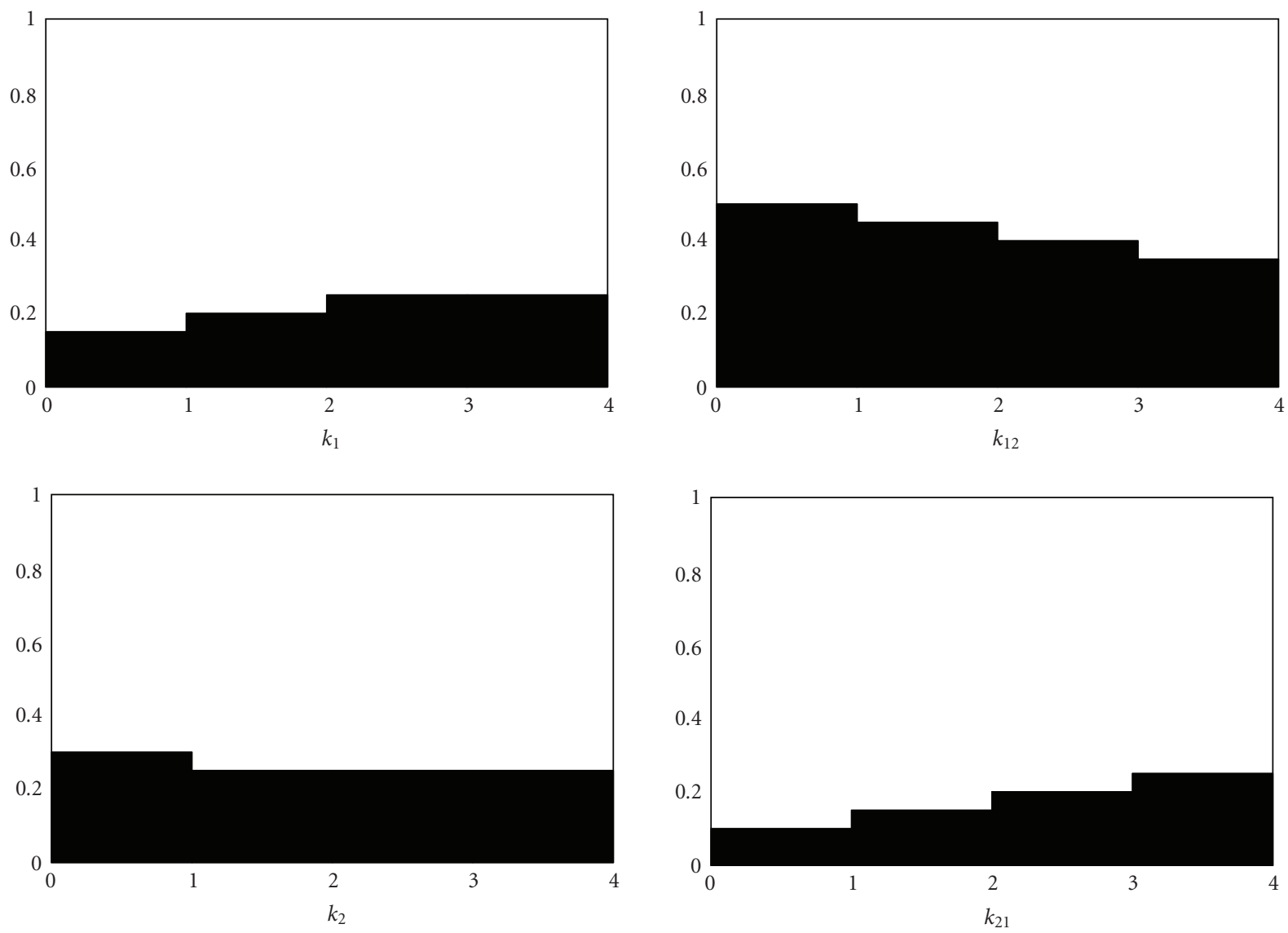

Figure 7: The variation of $k$ parameters due to the nonuniform traffic in the central cell.

of knapsacks are shared as much as the system's topology permits.

6.4. Relay's Coverage. We also considered the effect of the relays' coverage area on the total achieved throughput in the central cell. (There is a minimum SINR value required for each user to decode its data properly. A relay's coverage is defined as the area in the cell with an effective received SINR larger than the threshold value.) Note that if a user is outside of a relay's coverage, it means that the data of that user cannot be forwarded by that relay, meaning that its corresponding coefficient in (2) is zero. Two cases are investigated, namely, the limited coverage and the limited capacity scenarios. In the limited coverage case, the number of users distributed in the cell is not too much (50 users in our simulation). However, in the limited capacity case, 100 users were distributed in the cell boundary. The results for two different cases $\left(k_{1}=0.12\right.$ and $k_{1}=0.2$ ) are shown in Figure 9.

In the limited coverage case, by increasing the coverage area of the relays, the total achieved throughput is increased accordingly. This is because the number of users is not too much and they can be supported if there is a good coverage in the system. In other words, the relay's coverage is the limiting factor. However, in the limited capacity case, the number of users is so large that before increasing the coverage of relays to the maximum, the system reaches the maximum capacity.
This maximum capacity also depends on the value of $k_{1}$, as shown in Figure 9.

6.5. Complete System Simulation. Finally, a cellular network with a two-tier hexagonal cell configuration consisting of 19 cells is simulated. The wrap-around technique is also employed. Each cell has six sectors, where each sector is in cooperation with its neighboring sector in the neighboring cell. The pilot channel power is adjusted so that $40 \%$ of the users receive the pilot channel of the two BSs with an acceptable quality. The users' nonuniform spatial distribution is represented by a nonuniformity factor, $\chi$. This means that $(1-\chi) N$ users are distributed uniformly, and the rest of them are distributed in randomly located spots. For comparison, we consider three different systems. In system $\mathrm{I}$, there is no relaying in the system, and the routing scheme is based on a the pilot signal strength, so that the BS with the greatest $E_{c} / I_{0}$ is assigned to the user. In system II, the transmitter assignment is similar to that of system I; while we consider two relays in the system. Finally, system III uses our proposed joint rate allocation and routing scheme considering both BSs and the relays.

The effect of our proposed scheme on the normalized average achieved throughput is simulated while considering the nonuniformity factor. Two cases of user spatial distributions of $\chi=0.2$ and $\chi=0.5$ are considered. The simulations 

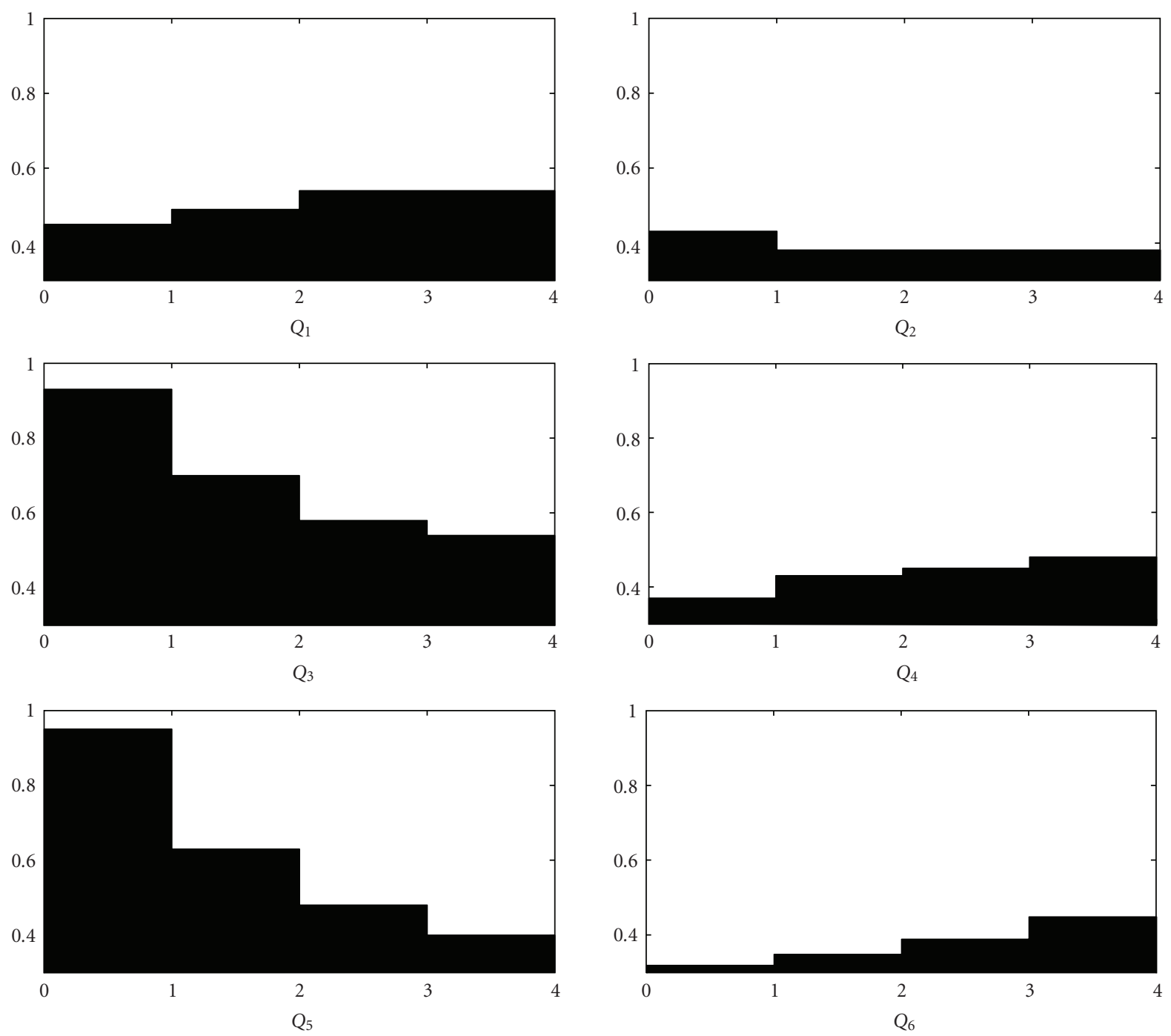

FIGURE 8: The variation of the $Q$ factors due to the adjustment of the $k$ parameters.

are executed 1000 times. For simplicity, the average achieved throughput of systems II and III is normalized by the average achieved throughput of system I. Figure 10 illustrates the normalized average achieved throughput versus the average number of users in each cell. As it can be seen, the average throughput of system III is larger than that of system II. The difference between the throughput gains of systems II and III indicates the gain due to using the MMKP capability to exploit the local information about the users locations and channel quality within the system. This gain is increased by the nonuniformity of the spatial distribution of the users.

6.6. Complexity Analysis. In order to study the run-time performance of the algorithm, we implemented it along with the optimal algorithm based on the branch and bound search using linear programming for the upper bound computation. We have performed experiments on an extensive set of the problem sets, where we used randomly generated MMKP instances for our tests. For each set of parameters $N$ and $M$, we run the algorithm ten times and tabulated the average of the achieved throughput and the execution time. Table 2 shows the percentage of the achieved throughput using our heuristic method compared to the value achieved in the optimal case. Moreover, the third column of Table 2 shows the required execution time in the heuristic method compared to that of the branch-and-bound method. It shows that the performance is really good for the large sets (greater than $95 \%$ most of the time), while the execution time is just a few percent of the time required for the optimal solution (less than 5\%).

\section{Conclusion}

A novel modeling for the joint BS/relay assignment, optimum rate allocation, and routing scheme was proposed and formulated under a single problem for the downlink multihop cellular networks. The concept of the capacity regions in the multihop cellular networks was then exploited to formulate the above problem as an MMKP to maximize the total achieved throughput in the system. The MMKP 


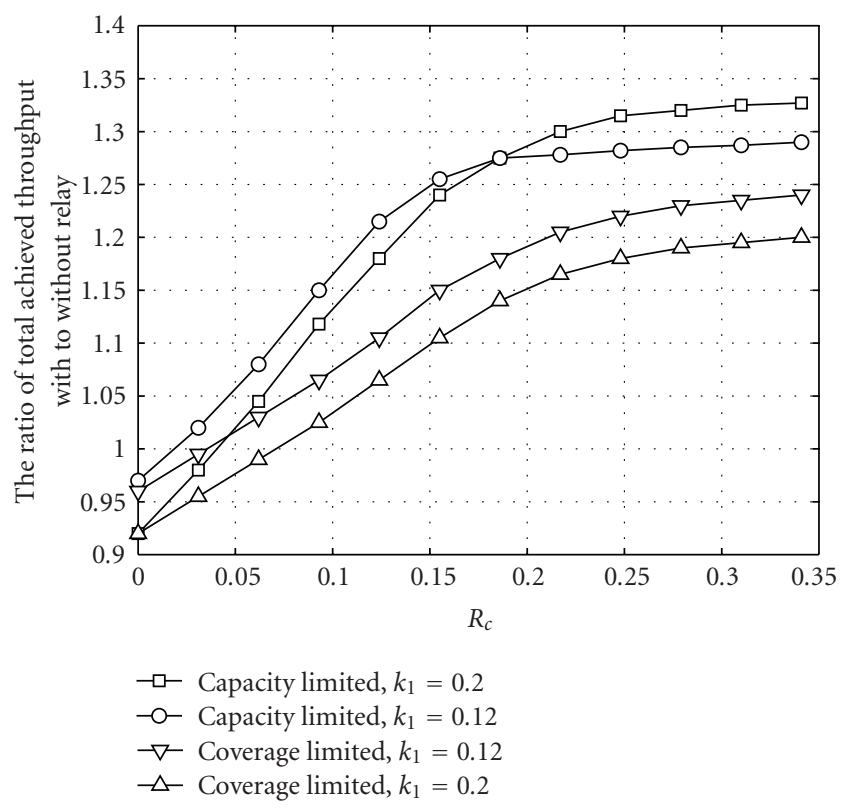

Figure 9: The total achieved throughput with and without relaying versus the relays' coverage.

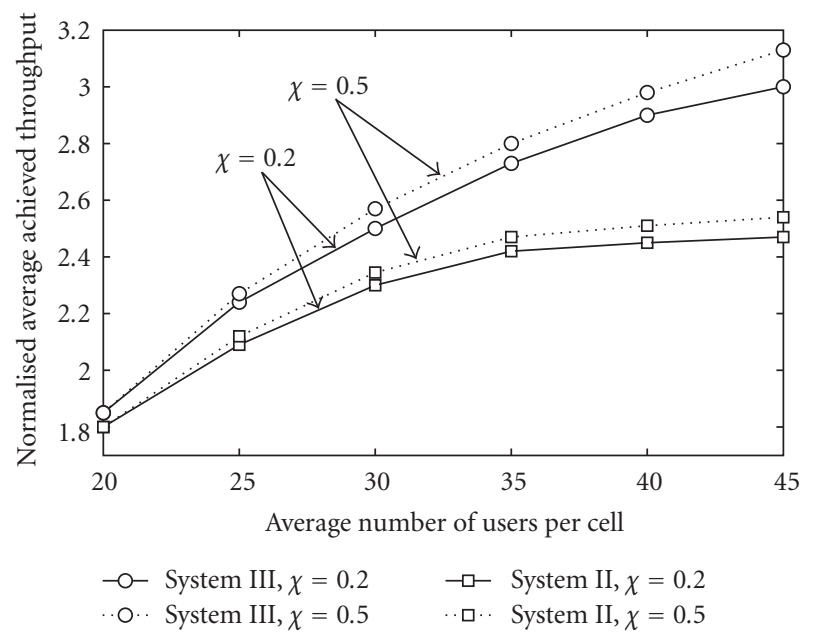

FIGURE 10: The normalized average achieved throughput of systems III and II versus the average number of users per cell.

algorithm based on the Lagrange multipliers was then modified to find a near-optimal solution with a linear complexity. The concept of the infeasibility factor was introduced to adjust the transmit powers of both the BSs and relays. In fact, the output of our algorithm is the joint rate allocation, routing scheme, and BS/relay assignment, which in conjunction with the proposed adaptive scheme leads to the implementation of the cell breathing via allocating the proper transmit powers to the BSs/relays.
TABle 2: Performance comparison of Branch-and-bound and a Heuristic algorithm in terms of total achieved throughput and execution time.

\begin{tabular}{lcc}
\hline$N$ & Value $\%$ & Time $\%$ \\
\hline 40 & 92.5 & 15.3 \\
70 & 95.6 & 4.2 \\
100 & 97.3 & 3.9 \\
130 & 98.1 & 2.7 \\
160 & 97.7 & 2.7 \\
190 & 98.1 & 2.9 \\
220 & 98.5 & 3.1 \\
250 & 98.7 & 3.1 \\
280 & 97.5 & 3.9 \\
310 & 97.4 & 3.0 \\
340 & 98.3 & 2.4 \\
370 & 99.3 & 1.9 \\
400 & 99.2 & 2.6 \\
\hline
\end{tabular}

\section{Appendices}

\section{A. Proof of Proposition 1}

The idea is to make the constraints independent of one another to be able to formulate the optimization problem using MMKP. We assume that $T_{w}=1$. First, let us consider the constraint (3). The BS 1 reserves $k_{1}$ portion of its total resource (time in TDS) for relay 1, meaning that the remaining resource that can be used for the direct transmission from BS 1 to the users can be written as $\sum_{j=5}^{N+4} \tau_{1 j} \leq 1-k_{1}$, which in turn implies that

$$
\sum_{j=5}^{N+4}\left(\frac{\tau_{3 j} R_{3 j}+\tau_{6 j} R_{4 j}}{R_{13}}\right) \leq k_{1} .
$$

On the other hand, allocating $k_{12} P_{\text {RLY }}$ of the total power of the relay 1 to forward the packets to the relay 2 leads to the following two constraints:

$$
\begin{aligned}
& \sum_{j=5}^{N+4}\left(\frac{\tau_{6 j} R_{4 j}}{R_{34}}\right) \leq k_{12}, \\
& \sum_{j=5}^{N+4}\left(\tau_{3 j}+\tau_{5 j}\right) \leq 1-k_{12},
\end{aligned}
$$

where (A.2) represents the part of relay 1 resource that is allocated to relay 2 (remember $\tau_{6 j}$ denotes the required time to forward the packets from relay 1 to relay 2), and (A.3) represents the remaining resource for relay 1 to serve the users (see Figure 3).

The constraints (A.2) along with (A.1) result in

$$
\sum_{j=5}^{N+4}\left(\frac{\tau_{3 j} R_{3 j}}{R_{13}}\right) \leq k_{1}-\left(\frac{k_{12} R_{34}}{R_{13}}\right) .
$$


Taking the same approach for relay 2, a counterpart constraint like the one for $\tau_{5 j}$ can be obtained for $\tau_{5 j}$ as

$$
\sum_{j=5}^{N+4}\left(\frac{\tau_{5 j} R_{3 j}}{R_{34}}\right) \leq k_{21} .
$$

There are three constraints on $\tau_{3 j}$ and $\tau_{5 j}$, namely, (A.3), (A.4), and (A.5). Therefore, we need to prove that (A.4) and (A.5) are the limiting constraints, meaning that they are more restrictive than (A.3). To do so, we use the result of the following proposition.

Proposition 3. Consider two constraints $\sum_{i=1}^{N} x_{i} \leq a$ and $\sum_{i=1}^{N} x_{i} y_{i} \leq b ; x_{i}, y_{i} \geq 0$, the second one is more limiting if and only if $1 / y_{i} \leq a / b$.

Proof. (Let $N=2$, then two constraints are two straight lines $x_{1}+x_{2} \leq a$ and $x_{1} y_{1}+x_{2} y_{2} \leq b$ in the two-dimensional Euclidean space. The valid values for each constraint are the area between its corresponding line and the lines $x_{1}=$ 0 and $x_{2}=0$. Therefore, the second constraint is more limiting when its area is a subset of the first one and this happens when $1 / y_{1} \leq a / b$ and $1 / y_{2} \leq a / b$. Similarly, in the three-dimensional space, $x_{1} y_{1}+x_{2} y_{2}+x_{3} y_{3} \leq b$ is more limiting than $x_{1}+x_{2}+x_{3} \leq a$ when the projection of this plane in each two-dimensional surface composed of $\left(x_{1}, x_{2}\right)$, $\left(x_{1}, x_{3}\right),\left(x_{1}, x_{3}\right)$ satisfies the conditions like the above, which leads to $1 / y_{i} \leq a / b$ for all $i \in\{1,2,3\}$. This idea can be generalized for $N$ parameters in $N$-dimensional Euclidean space accordingly.)

Proposition 4. The constraints in (A.4) and (A.5) are more restrictive than (A.3).

Proof. Combining (A.4) and (A.5) yields $\sum_{j=5}^{N+4}\left(\left(\tau_{3 j}+\right.\right.$ $\left.\left.\tau_{5 j}\right) R_{3 j}\right) \leq k_{1} R_{13}-k_{12} R_{34}+k_{21} R_{34}$. Using Proposition 3, it is just needed to show that based on the realistic values of the parameters, the following inequality holds

$$
\frac{1}{R_{3 j}} \leq \frac{\left(1-k_{12}\right)}{\left(k_{1} R_{13}-k_{12} R_{34}+k_{21} R_{34}\right)} \quad \forall j \in\{5, \ldots, N+4\},
$$

where $y_{i}=R_{3 j}, a=\left(1-k_{12}\right)$, and $b=\left(k_{1} R_{13}-k_{12} R_{34}+\right.$ $\left.k_{21} R_{34}\right)$. On the average, the distance of the relay 1 to any of its users is less than its distance to the relay 2 and BS (about half). Moreover, the total power of BSs are $L$ times that of the relays. These all lead to the result that $R_{3 j} \cong 16 R_{13} / L$, where 16 is the result of the power dissipation with exponent 4 , and the values of $k$ parameters are assumed in average to be 0.4 in our modeling. Therefore, the inequality in (A.6) evaluates as $1 /\left(16 R_{13} / L\right) \leq 0.6 / 0.4 R_{13}$, which considering the fact that $L \cong 5$ (Section 3 ) holds for all values of $j$. This means that constraints in (A.4) and (A.5) are more limiting than (A.3).

\section{B. Proof of Theorem 2}

Let us assume $X^{*}=\left\{x_{k j}^{*}\right\}$ is the output of the algorithm, and $Y^{*}=\left\{y_{k j}^{*}\right\}$ is the result of the globally optimum solution.
Based on the definition in the above algorithm, $T_{i}^{*}=$ $\sum_{j=1}^{N} \sum_{k=1}^{M+1} x_{k j}^{*} A_{i j k}$. Therefore, the total achieved throughput using the heuristic algorithm can be written as

$$
\begin{aligned}
\sum_{j=1}^{N} \sum_{k=1}^{M} x_{k j}^{*} \bar{R}_{j}= & \sum_{i=1}^{M} \sum_{j=1}^{N} \sum_{k=1}^{M+1} \lambda_{i} x_{k j}^{*} A_{i j k} \\
& +\sum_{j=1}^{N} \sum_{k=1}^{M} x_{k j}^{*} \bar{R}_{j}-\sum_{i=1}^{M} \sum_{j=1}^{N} \sum_{k=1}^{M+1} \lambda_{i} x_{k j}^{*} A_{i j k} \\
& +\sum_{k=1}^{M} \lambda_{i} T_{i}^{*}+\sum_{j=1}^{N} \sum_{k=1}^{M}\left(\bar{R}_{j}-\sum_{i=1}^{M} \lambda_{i} A_{i j k}\right) x_{k j}^{*},
\end{aligned}
$$

where (B.1) is derived using the fact that $A_{i j k}=0$ if $k=M+1$ for all $i, j$. For the optimal solution, $Y^{*}$, we can rewrite the same expression as in (B.1) as

$$
\sum_{j=1}^{N} \sum_{k=1}^{M} y_{k j}^{*} \bar{R}_{j}=\sum_{k=1}^{M} \lambda_{i} T_{i}^{*}+\sum_{j=1}^{N} \sum_{k=1}^{M}\left(\bar{R}_{j}-\sum_{i=1}^{M} \lambda_{i} A_{i j k}\right) y_{k j}^{*},
$$

where $T_{i}^{*}=\sum_{j=1}^{N} \sum_{k=1}^{M+1} y_{k j}^{*} A_{i j k}$. By definition, $T_{i}^{\prime} \leq C_{i}$ for all $i$. Therefore, the upper limit for (B.2) is

$$
\sum_{j=1}^{N} \sum_{k=1}^{M} y_{k j}^{*} \bar{R}_{j} \leq \sum_{k=1}^{M} \lambda_{i} C_{i}+\sum_{j=1}^{N} \sum_{k=1}^{M}\left(\bar{R}_{j}-\sum_{i=1}^{M} \lambda_{i} A_{i j k}\right) y_{k j}^{*}
$$

Using (B.1) and (B.3), the difference between the total achieved throughput using the suboptimal algorithm and the global optimal solution is

$$
\begin{aligned}
\sum_{j=1}^{N} \sum_{k=1}^{M} \bar{R}_{j}\left(y_{k j}^{*}-x_{k j}^{*}\right) \leq & \left\{\sum_{k=1}^{M} \lambda_{i}\left(C_{i}-T_{i}^{*}\right)\right. \\
& +\left\{\sum_{j=1}^{N} \sum_{k=1}^{M}\left(\bar{R}_{j}-\sum_{i=1}^{M} \lambda_{i} A_{i j k}\right) y_{k j}^{*}\right. \\
& \left.\left.-\sum_{j=1}^{N} \sum_{k=1}^{M}\left(\bar{R}_{j}-\sum_{i=1}^{M} \lambda_{i} A_{i j k}\right) x_{k j}^{*}\right\}\right\} .
\end{aligned}
$$

Let us denote the last term in (B.4) as $W=$ $\sum_{j=1}^{N} \sum_{k=1}^{M} \beta_{k j} y_{k j}^{*}-\sum_{j=1}^{N} \sum_{k=1}^{M} \beta_{k j} x_{k j}^{*}$, where $\beta_{k j}=\left(\bar{R}_{j}-\right.$ $\left.\sum_{i=1}^{M} \lambda_{i} A_{i j k}\right)$. We define the following sets $H_{1}=\left(X^{*} \cup Y^{*}\right)-$ $Y^{*}, H_{2}=\left(X^{*} \cup Y^{*}\right)-X^{*}$, and $H_{3}=\left(X^{*} \cap Y^{*}\right)$.

For the elements of $H_{3}$, it is clear that $W$ is equal to zero. For the elements of $H_{1}, \sum_{j=1}^{N} \sum_{k=1}^{M} \beta_{k j} y_{k j}^{*}=0$ and $\sum_{j=1}^{N} \sum_{k=1}^{M} \beta_{k j} x_{k j}^{*} \geq 0$, hence $W \leq 0$. As for the elements of $H_{2}, \sum_{j=1}^{N} \sum_{k=1}^{M} \beta_{k j} y_{k j}^{*} \leq 0$ (since $\left.\beta_{k j} \leq 0\right)$ and $\sum_{j=1}^{N} \sum_{k=1}^{M} \beta_{k j} x_{k j}^{*}=0$, thus, again $W \leq 0$. Therefore, in all cases, we have $W \leq 0$, which in conjunction with (B.4) leads to $\sum_{j=1}^{N} \sum_{k=1}^{M} \bar{R}_{j}\left(y_{k j}^{*}-x_{k j}^{*}\right) \leq \sum_{k=1}^{M} \lambda_{i}\left(C_{i}-T_{i}^{*}\right)=\sum_{k=1}^{M} \lambda_{i}\left(C_{i}-\right.$ $\left.\sum_{j=1}^{N} \sum_{k=1}^{M+1} x_{k j}^{*} A_{i j k}\right)$, which completes the proof. 


\section{Acknowledgment}

This work was supported by National Sciences and Engineering Research Council of Canada (NSERC).

\section{References}

[1] 3GPP, ODMA, http://www.3gpp.org/.

[2] Y.-D. Lin and Y.-C. Hsu, "Multihop cellular: a new architecture for wireless communications," in Proceedings of the 19th Annual Joint Conference of the IEEE Computer and Communications Societies (INFOCOM '00), vol. 3, pp. 1273-1282, Tel Aviv, Israel, March 2000.

[3] H. Wu, C. Qiao, S. De, and O. Tonguz, "Integrated cellular and ad hoc relaying systems: iCAR," IEEE Journal on Selected Areas in Communications, vol. 19, no. 10, pp. 2105-2115, 2001.

[4] M. DeFaria and E. S. Sousa, "Effect of intercell interference on the SNIR of a multihop cellular network," in Proceedings of the 61st IEEE Vehicular Technology Conference (VTC '05), vol. 5, pp. 3107-3111, Stockholm, Sweden, May-June 2005.

[5] R.-G. Cheng, S.-M. Cheng, and P. Lin, "Power-efficient routing mechanism for ODMA systems," IEEE Transactions on Vehicular Technology, vol. 55, no. 4, pp. 1311-1319, 2006.

[6] P. Lin, W.-R. Lai, and C.-H. Gan, "Modeling opportunity driven multiple access in UMTS," IEEE Transactions on Wireless Communications, vol. 3, no. 5, pp. 1669-1677, 2004.

[7] T. Rouse, S. McLaughlin, and I. Band, "Congestion-based routing strategies in multihop TDD-CDMA networks," IEEE Journal on Selected Areas in Communications, vol. 23, no. 3, pp. 668-681, 2005.

[8] V. Sreng, H. Yanikomeroglu, and D. Falconer, "Coverage enhancement through two-hop relaying in cellular radio systems," in Proceedings of the IEEE Wireless Communications and Networking Conference (WCNC '02), vol. 2, pp. 881-885, Orlando, Fla, USA, March 2002.

[9] T. J. Harrold and A. R. Nix, "Intelligent relaying for future personal communication systems," in Proceedings of the IEE Colloquium on Capacity and Range Enhancement Techniques for the Third Generation Mobile Communications and Beyond, pp. 1-5, London, UK, February 2000.

[10] J. Sun, "Uplink capacity enhancement in two-hop cellular networks with limited mobile relays," in Proceedings of the 15th IEEE Workshop on Local \& Metropolitan Area Networks (LANMAN '07), pp. 134-138, Princeton, NJ, USA, June 2007.

[11] E. S. Sousa, "Autonomous infrastructure wireless networks," in Proceedings of the 16th IST Mobile and Wireless Communications Summit, pp. 1-5, Budapest, Hungary, July 2007.

[12] K. Navaie and H. Yanikomeroglu, "Multi-user diversity in multi-hop cellular networks," in Proceedings of the Canadian Workshop on Information Theory (CWIT '05), Montreal, Canada, June 2005.

[13] K. M. Pepe and B. R. Vojcic, "Cellular multihop networks and the impact of routing on the SNIR and total power consumption," Journal of SPARTA, pp. 1-18, 2002.

[14] T. Rouse, S. McLaughlin, and H. Haas, "Coverage-capacity analysis of opportunity driven multiple access (ODMA) in UTRA TDD," in Proceedings of the 2nd International Conference on $3 G$ Mobile Communication Technology, pp. 252-256, London, UK, March 2001.

[15] T. Rouse, I. Band, and S. McLaughlin, "Capacity and power investigation of opportunity driven multiple access (ODMA) networks in TDD-CDMA based systems," in Proceedings of the
IEEE International Conference on Communications (ICC '02), vol. 5, pp. 3202-3206, New York, NY, USA, April-May 2002.

[16] H. Lee and C. C. Lee, "An integrated multihop cellular data network," in Proceedings of the 58th IEEE Vehicular Technology Conference (VTC '03), vol. 4, pp. 2232-2236, Orlando, Fla, USA, October 2003.

[17] 3GPP, "Opportunity driven multiple access," Tech. Rep. TR25.924 v1.0.0, 3GPP, Valbonne, France, 1999.

[18] M. Moser, "An algorithm for the multidimensional multiplechoice knapsack problem," IEICE Transactions on Fundamentals of Electronics, Communications and Computer Sciences, vol. 80, no. 3, pp. 582-589, 1997.

[19] M. Akbar, E. G. Manning, G. C. Shoja, and S. Khan, "Heuristic solutions for the multiple-choice multi-dimension knapsack problem," in Proceedings of International Conferences on Computational Science (ICCS '01), pp. 659-668, Stanford, Calif, USA, May 2001.

[20] W. Shih, "A branch and bound method for the multiconstraint knapsack problem," Journal of the Operational Research Society, vol. 30, no. 4, pp. 369-378, 1979.

[21] H. Everett III, "Generalized lagrange multiplier method for solving problems of optimum allocation of resources," Operations Research, vol. 11, no. 3, pp. 399-417, 1963. 